\title{
Schleswig-Holstein: CDU will Pflegekammer abschaffen
}

\section{Pflegeverbände sind entsetzt}

» Der Verband der Pflegedirektoren und -direktorinnen an den Universitätskliniken und medizinischen Hochschulen in Deutschland (VPU e.V.) ist entsetzt über die Ankündigung der CDU Schleswig-Holstein, die Pflegeberufekammer abschaffen zu wollen. Für den Managementverband bedeutet dies eine Missachtung der demokratischen Rechte der beruflich Pflegenden, die auch auf Bundesebene Folgen hätte.

Pflegekammern auf Landes- und Bundesebene stellen sicher, dass die Pflegeberufe ebenso wie andere Heilberufe, darunter Ärzte und Apotheker, gleich- und stimmberechtigt am politischen Entscheidungsprozess teilnehmen, die Interessen ihrer Mitglieder gebündelt vertreten, wichtige berufsspezifische Weichen stellen und die Weiterentwicklung der Pflegeberufe vorantreiben können.

Ein zentrales Ziel ist dabei, eine sachgerechte, professionelle Pflege - und damit eine möglichst hohe Versorgungsqualität für alle Bürgerinnen und Bürger - flächendeckend sicher zu stellen. „Es gibt keine Alternative zur Pflegekammer. Nicht in Schleswig-Holstein, nicht in den anderen Bundesländern und nicht auf Bundesebene. In einer demokratisch legitimierten Interessenvertretung der beruflich Pflegenden ist kein Platz für Arbeitgebervertreter", sagt Torsten Rantzsch, Vorstandsvorsitzender des VPU und Pflegedirektor am Universitätsklinikum Düsseldorf.

In ihrem Wahlprogramm zur Landtagswahl hatte die CDU angekündigt, die erst 2016 beschlossene Pflegeberufekammer durch eine freiwillige Pflegevereinigung zu ersetzen. Diese solle offen sein für Pflegende, Leistungserbringer und Verbände. Die Umsetzung soll nun im Koalitionsvertrag verankert werden. „Die Vorstellung, dass Arbeitnehmer und Arbeitgeber gemeinsam Qualitätsstandards für die professionelle Pflege entwickeln und sich gemeinsam für die strukturellen, wirtschaftlichen und politischen Forderungen der Pflegenden stark machen, ist absurd“, so Torsten Rantzsch.

Auch Andreas Westerfellhaus, Präsident des Deutschen Pflegerats e.V. (DPR), mahnt die möglichen Koalitionspartner in Schleswig-Holstein vor einem Stopp des Aufbaus der Pflegeberufekammer: „Nehmen Sie Abstand von Ihren Gedankenspielen, die sich im Aufbau befindliche Pflegeberufekammer in Schleswig-Holstein zu stoppen. Wir benötigen niemanden, der der Pflege den Weg ins 19. Jahrhundert zeigt, einem Zeitalter ohne Mitbestimmung und ohne Wertschätzung für die professionell Pflegenden." Ein Stopp des Kammer-Aufbaus hätte schwerwiegende Folgen für die Pflege in Schleswig-Holstein bis hin zur Gefährdung der Patientensicherheit.

www.vpuonline.de

\section{Hier steht eine Anzeige.}

\author{
然 Springer
}

